\title{
Eigenvalues of Killing Tensors and Separable Webs on Riemannian and Pseudo-Riemannian Manifolds`
}

\author{
Claudia CHANU and Giovanni RASTELLI
}

Dipartimento di Matematica, Università di Torino, Via Carlo Alberto 10, 10123 Torino, Italy

E-mail: claudiamaria.chanu@unito.it,giorast@tin.it

Received November 02, 2006, in final form January 16, 2007; Published online February 06, 2007

Original article is available at http://www.emis.de/journals/SIGMA/2007/021/

\begin{abstract}
Given a $n$-dimensional Riemannian manifold of arbitrary signature, we illustrate an algebraic method for constructing the coordinate webs separating the geodesic HamiltonJacobi equation by means of the eigenvalues of $m \leq n$ Killing two-tensors. Moreover, from the analysis of the eigenvalues, information about the possible symmetries of the web foliations arises. Three cases are examined: the orthogonal separation, the general separation, including non-orthogonal and isotropic coordinates, and the conformal separation, where Killing tensors are replaced by conformal Killing tensors. The method is illustrated by several examples and an application to the L-systems is provided.
\end{abstract}

Key words: variable separation; Hamilton-Jacobi equation; Killing tensors; (pseudo-)Riemannian manifolds

2000 Mathematics Subject Classification: 70H20; 70G45

\section{Introduction}

It is well-known that the separation of variables in the Hamilton-Jacobi equation of a natural Hamiltonian is characterized by the existence of sets of Killing 2-tensors $\left(\mathcal{K}_{m}\right)$ and Killing vectors $\left(D_{r}\right)$ with suitable properties $[17,4,6]$. The aim of this paper is to show some geometrical properties of a separable web (i.e., a set of foliations of coordinate hypersurfaces) by means of the analysis of $D_{r}$ and $\mathcal{K}_{m}$; in particular, we provide an algebraic method for determining the equations of the separable coordinate hypersurfaces. For instance, it is known that on a twodimensional manifold the separation is characterized by a Killing tensor $\mathbf{K}$ with pointwise simple eigenvalues $\left(\lambda^{1}, \lambda^{2}\right)$. Separable coordinates $\left(q^{1}, q^{2}\right)$ can be determined in two ways: (i) by setting $q^{1}=\lambda^{2}$ and $q^{2}=\lambda^{1}$ or, (ii) by integrating the eigenvectors $\left(\mathbf{E}_{1}, \mathbf{E}_{2}\right)$, which are orthogonal to the coordinate curves. In case (i) we need to assume that both eigenvalues are real independent functions (except for a closed subset of the configuration manifold at most); if the eigenvalues are not independent (for instance one of them is constant), then symmetries i.e., Killing vectors or ignorable coordinates, are present and the problem becomes even simpler. We remark that method (i) is purely algebraic: starting from $\mathbf{K}$, we have only to solve its characteristic equation.

In [11] we extended this analysis, for the orthogonal separation, to the $n$-dimensional Riemannian manifolds. For $n>2$ the eigenvalues of the Killing tensors do not define directly separable coordinates, however, we show how to construct rational functions of them which are constant on the separable coordinate hypersurfaces.

In the present paper we analyze the most general case of separation on a (pseudo) Riemannian manifold, without assumptions on the signature of the metric and on the orthogonality of the separable coordinates. In the following we shall refer to this kind of separation as "nonorthogonal separation" or "general separation". In non-orthogonal separation a fundamental role

${ }^{\star}$ This paper is a contribution to the Vadim Kuznetsov Memorial Issue "Integrable Systems and Related Topics". The full collection is available at http://www.emis.de/journals/SIGMA/kuznetsov.html 
is played by a particular $m$-dimensional space of Killing tensor $\mathcal{K}_{m}(m \leq n)$, whose properties relevant for our aims are recalled in Section 2. We illustrate an algebraic algorithmic method for constructing a set of intrinsically defined functions, that we call fundamental functions, starting from a special class of eigenvalues of $m$ independent Killing tensors of $\mathcal{K}_{m}$. The analysis of these functions allows us to detect two classes of symmetries (proper and conformal) of the Stäckel matrix associated with separation. When no proper symmetries occur, the fundamental functions are an effective tool for computing the equations of the separable coordinate hypersurfaces. Other algorithmic approaches for finding separable coordinates are already known in important particular cases, such as orthogonal separation in constant curvature manifolds with positive definite metric [16, 21], essentially based on the previous knowledge of all separable coordinates in these spaces (as generalized elliptic coordinates and their degenerations).

A further generalization considered here is the analysis of the case of the so-called orthogonal conformal separation, which deals with separable coordinates for the null-geodesic HJ-equation, or for a HJ-equation of a natural Hamiltonian with fixed value of the energy [7]. The orthogonal separable coordinates can be considered as a special case of this broader class of coordinates. In the intrinsic characterization of the orthogonal conformal separation, the Killing tensors are replaced by conformal Killing tensors with suitable properties. Following the same procedure of the "ordinary" separation, we are able to construct intrinsic functions allowing to deduce geometrical properties of the conformal separable coordinate hypersurfaces or to construct their equations.

In Section 2 we recall the basic intrinsic characterizations of the non-orthogonal separation on a Riemannian manifold in a form suitable for our needs. In Section 3 we describe our method and its application to a simple example. In Section 4, devoted to the orthogonal separation, we improve the analysis given in [11] and we show the links between eigenvalues of Killing tensors and proper or conformal symmetries for the associated coordinate systems. In the orthogonal case, by "proper symmetry" (resp., "conformal symmetry") of a coordinate system we mean that there are Killing vectors (resp., conformal Killing vectors) orthogonal to some foliations of the web. In Section 5, we see how the definitions of proper and conformal symmetries of the coordinates can be extended for the general separation and we generalize our results for the cases when non-orthogonal or null coordinates occur. In Section 6, we summarize the intrinsic characterization of the orthogonal conformal separation [7, 18] and we apply our algebraic method to the case of conformal separable orthogonal webs, showing how to detect conformal symmetries and to write the equation describing the foliations without conformal symmetries.

Each section is completed by illustrative examples: the spherical coordinates in $\mathbb{R}^{3}$ (Section 3 ), the L-systems [5], also known as Benenti systems [8, 9, 15] (Section 4), two non-orthogonal 4-dimensional coordinate systems (one of them with null coordinates) in Section 5, and the conformal separable coordinate system, known as tangent-spheres coordinates [20] (Section 6). Moreover, by applying our analysis to L-systems, we prove an interesting geometrical property of these systems: for $n>2$, none of the common eigenvectors of the associated Killing tensors is a proper conformal Killing vector.

\section{An outline of geodesic separation on Riemannian and pseudo-Riemannian manifolds}

We consider a $n$-dimensional Riemannian manifold $Q$ with contravariant metric $\mathbf{G}=\left(g^{i j}\right)$ of arbitrary signature and the corresponding geodesic Hamiltonian $G=\frac{1}{2} g^{i j} p_{i} p_{j}$. A relation of equivalence is defined among separable coordinate systems for $G[1,2]$ such that in each class there are the particular coordinate systems described in Theorem 1 below. We recall 
that a regular square matrix $\left(\varphi_{j}^{(i)}\right)$ is a Stäckel matrix if any element $\varphi_{j}^{(i)}$ is a function of the coordinate $q^{j}$ only.

Theorem 1 ([1, 2]). In an equivalence class of separable coordinates there exists a standard coordinate system $\left(q^{\hat{a}}, q^{\bar{a}}, q^{\alpha}\right)$ such that (i) The metric tensor has the standard form

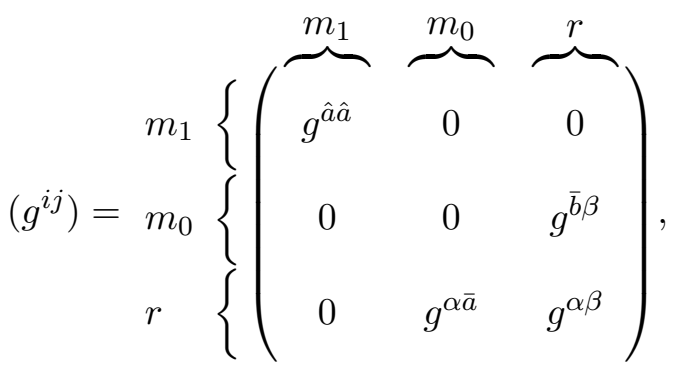

where the coordinates $\left(q^{\alpha}\right)\left(\alpha=m_{0}+m_{1}+1, \ldots, n\right)$ are ignorable, $\partial_{\alpha} g^{i j}=0(i, j=1, \ldots, n)$. (ii) The non-vanishing metric components have the form

$$
\begin{array}{lll}
g^{\hat{a} \hat{a}}=\varphi_{(m)}^{\hat{a}}, & & a=1, \ldots, m_{1}+m_{0}, \\
g^{\bar{a} \beta}=\theta_{\bar{a}}^{\beta} \varphi_{(m)}^{\bar{a}}, & & \hat{a}=1, \ldots, m_{1}, \\
g^{\alpha \beta}=\eta_{a}^{\alpha \beta} \varphi_{(m)}^{a}, & & \alpha, \beta=m_{1}+1, \ldots, m_{1}+m_{0}, \\
&
\end{array}
$$

where $\theta_{\bar{a}}^{\beta}$ and $\eta_{a}^{\alpha \beta}$ are functions of the coordinate corresponding to the lower index only and $\left(\varphi_{(m)}^{a}\right)=\left(\varphi_{(m)}^{\hat{a}}, \varphi_{(m)}^{\bar{a}}\right), m=m_{0}+m_{1}$, is a row of the inverse of a $m \times m$ Stäckel matrix $\mathbf{S}$ in the coordinates $\left(q^{a}\right)$.

Theorem 2 ([4]). The geodesic Hamiltonian $G$ is separable if and only if there exists a pair $\left(D_{r}, \mathcal{K}_{m}\right)$, called separable Killing algebra, such that: a) $D_{r}$ is a r-dimensional Abelian algebra of Killing vectors spanning a regular distribution $\Delta$ of rank $r$ such that $I=\Delta \cap \Delta^{\perp}$ has constant rank $m_{0}$. b) $\mathcal{K}_{m}$ is a m-dimensional space of Killing tensors, generated by $m$ independent tensors $\left(\mathbf{K}_{a}\right)$, with $m$ common eigenvectors orthogonal to $D_{r}$ which are normal (i.e., orthogonally integrable) and associated with real eigenvalues. c) $\mathcal{K}_{m}$ is $D_{r}$-invariant. d) For $m_{0}>1$, $d(\mathbf{K}$. $\left.d g^{\alpha \beta}\right)=0$ for any $\mathbf{K} \in \mathcal{K}_{m}$, where $g^{\alpha \beta}=\mathbf{X}_{\alpha} \cdot \mathbf{X}_{\beta}$ for any basis $\left(\mathbf{X}_{\alpha}\right)$ of $D_{r}$.

By "Killing tensor" (KT) we mean a symmetric two-tensor $\mathbf{K}$ such that $[\mathbf{K}, \mathbf{G}]=0$, where $[\cdot, \cdot]$ denotes the Lie-Schouten brackets. With a separable Killing algebra we associate an important kind of coordinates in the following way:

Definition 1. Let $\left(D_{r}, \mathcal{K}_{m}\right)$ be a separable Killing algebra and $\left(\mathbf{E}_{a}\right)$ be the $m$ normal common eigenvectors $\left(\mathbf{E}_{a}\right)$ of the elements of $\mathcal{K}_{m}$. The coordinates $\left(q^{a}, q^{\alpha}\right)$ are adapted coordinates of $\left(D_{r}, \mathcal{K}_{m}\right)$ if the coordinate hypersurfaces of $q^{a}$ are the integral manifolds orthogonal to each $\mathbf{E}_{a}$ (or equivalently, the differentials $d q^{a}$ are common eigenforms of $\mathcal{K}_{m}$ ) and the vector fields $\partial_{\alpha}$ form a basis of $D_{r}$ (i.e., the $q^{\alpha}$ are the affine parameters of a given basis $\mathbf{X}_{\alpha}$ of $D_{r}$ with zero values on a chosen integral manifold of $\left.\Delta^{\perp}\right)$; the $m$ orthogonal coordinates $\left(q^{a}\right)$ are said to be essential. The $m_{0}$ essential coordinates $\left(q^{\bar{a}}\right)$ such that $g^{\bar{a} \bar{a}}=0$ are called isotropic or null coordinates.

The integrability of the distribution $\Delta^{\perp}$ is a consequence of the hypotheses of Theorem 2 [4]. We remark that if $Q$ is a proper Riemannian manifold (or if the coordinates are orthogonal) there are no isotropic coordinates. 
Theorem $3([4])$. Let $\left(q^{i}\right)=\left(q^{\hat{a}}, q^{\bar{a}}, q^{\alpha}\right)$ be an adapted coordinate system of a separable Killing algebra $\left(D_{r}, \mathcal{K}_{m}\right)$. Then, (i) The coordinates $\left(q^{i}\right)$ are standard separable coordinates and each tensor $\mathbf{K} \in \mathcal{K}_{m}$ assumes the standard form

$$
\left(K^{i j}\right)=\left(\begin{array}{ccc}
\lambda^{\hat{a}} g^{\hat{a} \hat{a}} & 0 & 0 \\
0 & 0 & \lambda^{\bar{b}} g^{\bar{b} \beta} \\
0 & \lambda^{\bar{a}} g^{\alpha \bar{a}} & K^{\alpha \beta}
\end{array}\right) .
$$

(ii) Given a basis $\left(\mathbf{K}_{a}\right)$ of $\mathcal{K}_{m}$, the non-vanishing components of each $\mathbf{K}_{b}(b=1, \ldots, m)$ assume the form

$$
\begin{aligned}
& K_{b}^{\hat{a} \hat{a}}=\varphi_{(b)}^{\hat{a}}, \quad a=1, \ldots, m, \quad b=1, \ldots, m, \\
& K_{b}^{\bar{a} \beta}=\theta_{\bar{a}}^{\beta} \varphi_{(b)}^{\bar{a}}, \quad \hat{a}=1, \ldots, m_{1}, \quad \bar{a}=m_{1}+1, \ldots, m, \\
& K_{b}^{\alpha \beta}=\eta_{a}^{\alpha \beta} \varphi_{(b)}^{a}, \quad \alpha, \beta=m+1, \ldots, n,
\end{aligned}
$$

where $\left(\varphi_{(b)}^{a}\right)$ is a row of $\mathbf{S}^{-1}$.

Remark 1. The functions $\left(\lambda^{a}\right)$ are the eigenvalues of $\mathbf{K}$ corresponding to the common eigenvectors of all tensors in $\mathcal{K}_{m}$ and satisfy the following intrinsic Killing equations:

$$
\begin{aligned}
& \partial_{a} \lambda^{b}=\left(\lambda^{a}-\lambda^{b}\right) \partial_{a} \ln \varphi_{(m)}^{b}, \\
& \partial_{a} K^{\alpha \beta}=\lambda^{a} \partial_{a} g^{\alpha \beta}, \quad a=(\hat{a}, \bar{a}), \quad a, b=1, \ldots, m, \quad j=1, \ldots, n \text {. } \\
& \partial_{\alpha} \lambda^{j}=0 \text {, }
\end{aligned}
$$

The geometric realization of an equivalence class of separable coordinates is called separable Killing web [4]:

Definition 2. A separable Killing web is a triple $\left(\mathcal{S}_{m}, D_{r}, \mathbf{K}\right)$, where (i) $\mathcal{S}_{m}=\left(S^{a}\right)$ is a set of $m$ foliations of hypersurfaces pairwise transversal and orthogonal; (ii) $D_{r}$ is a $r$-dimensional Abelian algebra of Killing vectors tangent to each foliation $S^{a}$; (iii) $\mathbf{K}$ is a $D_{r}$-invariant Killing tensor with $m$ eigenvectors $\left(\mathbf{E}_{a}\right)$ associated with $m$ pointwise distinct real eigenvalues $\left(\lambda^{a}\right)$ and orthogonal to the foliations $\left(S^{a}\right)$. The $\mathrm{KT} \mathbf{K}$ is called characteristic tensor of the web.

Remark 2. The existence of a separable Killing web is equivalent to the existence of a separable Killing algebra, or of separable coordinates for $G$. Indeed, a separable Killing web $\left(\mathcal{S}_{m}, D_{r}, \mathbf{K}\right)$ gives rise to a standard separable coordinate system $\left(q^{a}, q^{\alpha}\right)$ such that the coordinate hypersurfaces $q^{a}=$ constant are leaves of $S^{a}$ and the vector fields $\left(\partial_{\alpha}\right)$ associated with $q^{\alpha}$ form a basis of $D_{r}$.

From Definitions 1 and 2, it follows that essential coordinates only are associated with the eigenvectors of Killing tensors, that is with the foliations $\left(S^{a}\right)$ of a separable Killing web. Therefore, in the following sections we restrict our attention to the essential coordinates. In Example 3 we show a separable Killing web, the corresponding separable Killing algebra and the Stäckel matrix in the adapted coordinates for the case $n=4, m=2$ and $m_{0}=0$.

\section{The method of the eigenvalues}

In order to clarify the exposition, we collect in this section the results proved in Sections 4-6. We recall that 
Definition 3. A vector field $\mathbf{X}$ is said to be a conformal Killing vector (CKV) if there exists a function $F$ such that

$$
[\mathbf{X}, \mathbf{G}]=\mathcal{L}_{\mathbf{X}} \mathbf{G}=F \mathbf{G}
$$

where $[\cdot, \cdot]$ is the Schouten bracket and $\mathcal{L}$ the Lie-derivative. If $F=0, \mathbf{X}$ is a Killing vector $(\mathrm{KV})$ and if $F \neq 0$ we call $\mathbf{X}$ a proper $\mathrm{CKV}$.

Remark 3. A coordinate $q^{i}$ is ignorable if and only if $\partial_{i}$ is a Killing vector. Moreover, in an orthogonal system, if $\partial_{i}$ is proportional to a KV (i.e., there is a function $f$ such that $f \partial_{i}$ is a KV) then $q^{i}$ is ignorable up to a rescaling $\tilde{q}^{i}=\tilde{q}^{i}\left(q^{i}\right)$.

Note 1. Here and in the following, for any matrix $\mathbf{A}=\left(a_{j}^{i}\right)$ the lower index is the row-index and the upper one is the column index. Moreover, we shall denote by $\mathbf{A}_{j}^{i}$ the submatrix of $\mathbf{A}$ obtained by eliminating the $j$-th row and the $i$-th column.

Step 1. Construction of the Fundamental functions.

Let $\left(D_{r}, \mathcal{K}_{m}\right)$ be a separable Killing algebra associated with a separable web $\left(D_{r}, \mathcal{S}_{m}\right)$ and let $\left(\mathbf{K}_{1}, \ldots, \mathbf{K}_{m}=\mathbf{G}\right)$ be a basis of $\mathcal{K}_{m}$.

i) We determine the essential eigenvalues of $\left(\mathbf{K}_{a}\right)$ associated with the common essential eigenvectors $\left(\mathbf{E}_{1}, \ldots, \mathbf{E}_{m}\right)$ orthogonal to $D_{r}$.

ii) We construct the regular (see Remark 10) $m \times m$ matrix $\Lambda=\left(\lambda_{a}^{b}\right)$ of the essential eigenvalues of $\mathbf{K}_{a} \in \mathcal{K}_{m}$ ordered as follows: $\lambda_{a}^{b}$ is the eigenvalue of $\mathbf{K}_{a}$ associated with the common eigenvector $\mathbf{E}_{b}$.

We remark that for the construction of $\Lambda$ we have to order properly the eigenvalues of each $\mathrm{KT}$ and, to do that, we need to compute the eigenvectors. However, our further analysis is based only upon the matrix $\Lambda$ of the eigenvalues and no integration is needed.

iii) For $a, b, c=1, \ldots, m$ we consider the intrinsic ratios

$$
f_{a}^{b c}=\frac{\operatorname{det} \Lambda_{b}^{a}}{\operatorname{det} \Lambda_{c}^{a}}
$$

that we call fundamental functions. They are well-defined functions only if $\operatorname{det} \Lambda_{c}^{a}$ is not identically zero. Moreover, since $f_{a}^{b c}=1 / f_{a}^{c b}$ and $f_{a}^{b b}$ is equal to 1 or everywhere undefined, in the following we shall assume $b>c$.

\section{Step 2. Analysis.}

Let us fix an index $a$. Due to the regularity of $\Lambda$, at least one fundamental function (3.1) is well defined (Proposition 3). By examining the functions $f_{a}^{b c}$ written in an arbitrary coordinate system, we can easily detect symmetries of the foliation $S^{a} \in \mathcal{S}_{m}$; if $S^{a}$ has no symmetries we obtain the equation of the foliation $S^{a}$. Indeed, two different and mutually exclusive cases occur:

iv) There exist indices $c$ and $b$ such that the function $f_{a}^{b c}$ is not identically constant. In this case, in a neighborhood of each point $P_{0}$ such that $d f_{a}^{b c}\left(P_{0}\right) \neq 0$, equation

$$
f_{a}^{b c}=f_{a}^{b c}\left(P_{0}\right)
$$

defines a hypersurface containing $P_{0}$ and orthogonal to the eigenvector $\mathbf{E}_{a}$ (i.e., a hypersurface of $S^{a}$ ). Hence, equations

$$
f_{a}^{b c}=k,
$$

for suitable values of $k \in \mathbb{R}$, describe the foliation $S^{a}$ (see Theorems 5 and 8 , for the orthogonal and the general case respectively). 
v) For the fixed index $a$ all functions $f_{a}^{b c}$ constructed in STEP 1 are constant or undefined. Then, special properties of the adapted coordinates of $\left(D_{r}, \mathcal{K}_{m}\right)$ hold. Let $\left(q^{1}, \ldots, q^{m}\right)$ be the essential coordinates adapted to the foliations $\left(S^{1}, \ldots, S^{m}\right)$. Up to a reparameterization of $q^{a}$, the Stäckel matrix $\mathbf{S}=\left(\varphi_{c}^{(b)}\right)$ and its inverse matrix $\mathbf{S}^{-1}=\left(\varphi_{(c)}^{b}\right)=\left(\lambda_{c}^{b} \varphi_{(m)}^{b}\right)$ do not depend on $q^{a}$ (see Theorem 7 (ii)). We call the vector field $\partial_{a}$ a Stäckel symmetry (see Section 5 for further details).

We remark that we do not need to distinguish between isotropic and non-isotropic coordinates. Moreover, if we consider the orthogonal separation (i.e., when $m=n, D_{r}=0$ and all coordinates are essential) then we are able to test if a foliation $S^{a}$ is orthogonal to a Killing vector or to a conformal Killing vector, by examining the fundamental functions. Indeed, the following properties hold:

vi) All fundamental functions $f_{a}^{b c}$ are constant or undefined if and only if the eigenvector $\mathbf{E}_{a}$ is proportional to a Killing vector i.e., the associated adapted coordinate $q^{a}$ is (up to a reparameterization) ignorable (Theorem 4 (ii)).

vii) All fundamental functions $f_{a}^{b c}$ with $c<b<n(n>2)$ are constant or undefined if and only if the corresponding eigenvector $\mathbf{E}_{a}$ is proportional to a conformal Killing vector (Theorem 4 (i)).

Remark 4. Also for the general separation, properties analogous to items vi) and vii) hold. Item vi) is in fact a special case of the general situation described in item v), holding for orthogonal coordinates. Indeed, due to Remark 3 and to equations $(2.5)_{1}$, we have that $\mathbf{E}_{a}$ is proportional to a Killing vector if and only if the Stäckel matrix and its inverse do not depend on the corresponding coordinate $q^{a}$. The property stated in item vii) can be extended to general separable coordinates as follows (Theorem 7 (i)): All functions $f_{a}^{b c}$ with $c<b<m$ $(m>2)$ are constant or undefined if and only if there exists a function $F$ such that (up to a reparameterization of $\left.q^{a}\right) \partial_{a} \varphi_{(m)}^{b}=F \varphi_{(m)}^{b}$ for all $b=1, \ldots, m$. Then, we call $\partial_{a}$ a conformal Stäckel symmetry (see Section 5 for further details).

Remark 5. For $m=2$, we have only two fundamental functions: $f_{1}^{21}=\lambda^{2}$ and $f_{2}^{21}=\lambda^{1}$ i.e., the eigenvalues of the characteristic tensor.

We show how the method works in the following simple but illustrative example.

Example 1. Let us consider in $\mathbb{R}^{3}$ the spherical coordinates centered at a point $O$, with axis $\omega$ passing through $O$ and parallel to a unit vector $\mathbf{n}$. It is well known that they are orthogonal separable coordinates for the geodesic Hamiltonian. Thus, we have $m=n=3$ and all coordinates are essential. A basis of $\mathcal{K}_{3}$ is

$$
\mathbf{K}_{1}=r^{2} \mathbf{G}-\mathbf{r} \otimes \mathbf{r}, \quad \mathbf{K}_{2}=(\mathbf{n} \times \mathbf{r}) \otimes(\mathbf{n} \times \mathbf{r}), \quad \mathbf{K}_{3}=\mathbf{G},
$$

where $\mathbf{r}$ is the position vector with respect to $O$ and $r=\|\mathbf{r}\|$. The common eigenvectors are

$$
\mathbf{E}_{1}=\mathbf{r}, \quad \mathbf{E}_{2}=\mathbf{r} \times(\mathbf{n} \times \mathbf{r}), \quad \mathbf{E}_{3}=\mathbf{n} \times \mathbf{r},
$$

which are orthogonal to the foliation $S^{1}$ of the spheres centered at $O$, to the foliation $S^{2}$ of the circular cones with vertex $O$ and axis $\mathbf{n}$ and to the foliation $S^{3}$ of the meridian half-planes issued from $\omega$, respectively. The matrix $\Lambda$ of the eigenvalues of $\mathbf{K}_{a}$ is

$$
\Lambda=\left(\begin{array}{ccc}
0 & r^{2} & r^{2} \\
0 & 0 & \|\mathbf{n} \times \mathbf{r}\|^{2} \\
1 & 1 & 1
\end{array}\right) .
$$


By computing $\operatorname{det} \Lambda_{b}^{a}$ for $a, b=1, \ldots, 3$, we get that the non vanishing ones are

$$
\Lambda_{1}^{1}=\Lambda_{1}^{2}=-\|\mathbf{n} \times \mathbf{r}\|^{2}, \quad \Lambda_{3}^{1}=r^{2}\|\mathbf{n} \times \mathbf{r}\|^{2}, \quad \Lambda_{2}^{2}=\Lambda_{2}^{3}=-r^{2} .
$$

The fundamental functions (3.1) are summarized in the following table (n.d. means that the denominator vanishes identically and the function is not defined)

\begin{tabular}{|l|c|c|c|}
\hline & $c=1, b=2$ & $c=1, b=3$ & $c=2, b=3$ \\
\hline$a=1$ & $f_{1}^{21}=0$ & $f_{1}^{31}=-r^{2}$ & $f_{1}^{32}$ n.d. \\
\hline$a=2$ & $f_{2}^{21}=\frac{r^{2}}{\|\mathbf{n} \times \mathbf{r}\|^{2}}$ & $f_{2}^{31}=0$ & $f_{2}^{32}=0$ \\
\hline$a=3$ & $f_{3}^{21}$ n.d. & $f_{3}^{31}=0$ & $f_{3}^{32}$ n.d. \\
\hline
\end{tabular}

For $a=1$, the function $f_{1}^{31}=-r^{2}$ is constant on the hypersurfaces of $S^{1}$ and equation $f_{1}^{31}=k$, for real negative values of $k$, describes all the spheres of $S^{1}$. According to the fact that $\mathbf{E}_{1}=\mathbf{r}$ is a $\operatorname{CKV}\left(\mathcal{L}_{\mathbf{r}} \mathbf{G}=-2 \mathbf{G}\right)$, we have that for all $c<b<3$ all functions $f_{1}^{b c}$ are constant or undefined.

For $a=2$, the level sets of the non-constant function $f_{2}^{21}=r^{2}\|\mathbf{n} \times \mathbf{r}\|^{-2}$ are the surfaces of $S^{2}$ and, since the upper indices are both $<3$, the eigenvector $\mathbf{E}_{2}$ is not proportional to a CKV.

For $a=3$, since for any $b$ and $c, f_{3}^{b c}$ is undefined or identically constant, we have that $\mathbf{E}_{3}$ is a Killing vector (the rotation around the axis $\omega$ ) and the corresponding coordinate $q^{3}$ (the rotational angle) is ignorable.

\section{Orthogonal separable webs}

We consider a $n$-dimensional Riemannian manifold $Q$ with positive definite metric $\mathbf{G}$ and the corresponding geodesic Hamiltonian $G=\frac{1}{2} g^{i j} p_{i} p_{j}$. We suppose that $G$ is orthogonally separable. We adapt the general results of Section 2 to the case $m=n, m_{0}=0$. Thus, some of the geometric structures introduced in Section 2 are simplified. The separable Killing web (Definition 2) is replaced by the orthogonal separable web $\left(\mathcal{S}_{n}, \mathbf{K}\right)$, that is a set of $n$ pairwise orthogonal foliations $\mathcal{S}_{n}=\left(S^{i}\right)$ orthogonal to the eigenvectors of the Killing tensor $\mathbf{K}$ with simple eigenvalues (the characteristic tensor of the web). In the orthogonal context, the linear space $D_{r}$ of Killing vectors disappears and the $n$-dimensional space of Killing tensors $\mathcal{K}_{n}$ associated with $\mathcal{S}_{n}$ is called Killing-Stäckel algebra (KS-algebra) or Killing-Stäckel space. All Killing tensors of $\mathcal{K}_{n}$ have common normal (i.e., orthogonally integrable) eigenvectors $\left(\mathbf{E}_{i}\right)$, the integral manifolds orthogonal to $\mathbf{E}_{i}$ are the leaves of $S^{i}$ and all coordinates are essential. We denote by $\left(\mathbf{K}_{j}\right)$ a basis of $\mathcal{K}_{n}$ with $\mathbf{K}_{n}=\mathbf{G}$. Adapting Theorem 3 to the orthogonal separation, we get

Proposition 1. Let $\left(q^{i}\right)$ be a coordinate system adapted to the KS-algebra $\mathcal{K}_{n}$. Then, (i) the $\left(q^{i}\right)$ are orthogonal separable coordinates for $G$. (ii) Given a basis $\left(\mathbf{K}_{j}\right)$ of $\mathcal{K}_{n}$, we have

$$
\mathbf{K}_{j}=\sum_{i} \lambda_{j}^{i} g^{i i} \mathbf{E}_{i} \otimes \mathbf{E}_{i}=\sum_{i} K_{j}^{i i} \mathbf{E}_{i} \otimes \mathbf{E}_{i}, \quad \forall j=1, \ldots, n,
$$

where $\mathbf{E}_{i}$ are common eigenvectors of $\mathbf{K}_{j}$ and $\lambda_{j}^{i}$ are the corresponding eigenvalues.

We call $\mathbf{S}^{-1}=\left(\varphi_{(j)}^{i}\right)$ the regular $n \times n$ matrix of the components of $\left(\mathbf{K}_{j}\right)$ :

$$
\varphi_{(j)}^{i}=K_{j}^{i i}=\lambda_{j}^{i} g^{i i}, \quad \varphi_{(n)}^{i}=K_{n}^{i i}=g^{i i} .
$$


As in the general case (see equation (2.4)), its inverse matrix $\mathbf{S}=\left(\varphi_{i}^{(j)}\right)$ is a Stäckel matrix. Moreover, we consider the invariant $n \times n$ matrix $\Lambda=\left(\lambda_{j}^{i}\right)$ of the eigenvalues of a basis of $\mathcal{K}_{n}$, introduced in Section 3. Theorems 4 and 5 below give a complete algebraic characterization of orthogonal separable webs in terms of eigenvalues of associated Killing-Stäckel spaces, improving considerably the analysis contained in [11] and providing a rigorous proof of the method illustrated in Section 3 for the orthogonal separation.

Proposition 2. For any fixed index $i=1, \ldots, n$ the fundamental functions

$$
f_{i}^{j h}=\frac{\operatorname{det} \Lambda_{j}^{i}}{\operatorname{det} \Lambda_{h}^{i}}
$$

when well-defined, depend on $q^{i}$ only: in particular we have

$$
f_{i}^{j h}=(-1)^{h+j} \frac{\varphi_{i}^{(j)}}{\varphi_{i}^{(h)}} .
$$

Proof. We have the following relations between $\mathbf{S}^{-1}$ and $\Lambda$ :

$$
\operatorname{det} \mathbf{S}^{-1}=\operatorname{det} \Lambda \prod_{i} g^{i i}, \quad \operatorname{det}\left(\mathbf{S}^{-1}\right)_{h}^{k}=\frac{\operatorname{det} \Lambda_{h}^{k}}{g^{k k}} \prod_{i} g^{i i} .
$$

By definition of inverse matrix and by (4.4), we see that each element of $\mathbf{S}$ has the following expression

$$
\varphi_{i}^{(j)}=(-1)^{i+j} \frac{\operatorname{det}\left(\mathbf{S}^{-1}\right)_{j}^{i}}{\operatorname{det} \mathbf{S}^{-1}}=(-1)^{i+j} \frac{\operatorname{det} \Lambda_{j}^{i}}{g^{i i} \operatorname{det} \Lambda} .
$$

Hence, by (4.2), equation (4.3) holds and the fundamental functions (4.2) depend on the coordinate $q^{i}$ only.

Proposition 3. For any index $i$ there exist indices $h$ and $j$ with $h<j$ such that the function (4.2) is well-defined.

Proof. Since $\operatorname{det} \Lambda \neq 0$, for each $i$ there exists an index $h_{0}$ such that $\operatorname{det} \Lambda_{h_{0}}^{i} \neq 0$. If $h_{0}<n$, then $f_{i}^{j h_{0}}$ is well-defined for any $j>h_{0}$. If $h_{0}=n$, then there exists an index $h<n$ such that $\operatorname{det} \Lambda_{h}^{i} \neq 0$. Indeed, the $n \times(n-1)$ matrix $\Lambda^{i}$ obtained from $\Lambda$ by eliminating the $i$-th column has rank $n-1$. Moreover, being $\operatorname{det} \Lambda_{n}^{i} \neq 0$, the first $n-1$ lines are independent i.e., form a basis of a $(n-1)$-dimensional linear space. Since the last row is different from zero (all its elements are equal to 1 ), there exists a basis made of the last row and other $n-2$ rows of $\Lambda^{i}$. This means that there exists $h<n$ such that $\operatorname{det} \Lambda_{h}^{i} \neq 0$. Hence, for any index $i$, at least one function $f_{i}^{j h}$ with $j>h$ is well defined.

From the Definition 3 of CKV, we get the following lemma

Lemma 1. The vector field $\mathbf{X}=f\left(q^{1}, \ldots, q^{n}\right) \partial_{i}$ is a $C K V$ if and only if (i) $f$ depends on $q^{i}$ only; (ii) there exists a function $F$ such that

$$
\partial_{i} \ln g^{j j}=F, \quad j \neq i, \quad \partial_{i} \ln g^{i i}=F+2 \partial_{i} \ln f .
$$

In particular if $F=0$, then $\mathbf{X}$ is a $K V$. 
Remark 6. By (4.5) it follows that $\partial_{i} g^{h h}=\partial_{i} g^{j j}$ for all $h, j$ both different from $i$. Moreover, due to item (i) of Lemma 1 the coordinate $q^{i}$ can always be rescaled in order to have $\mathbf{X}=\partial_{i}$. This means that if $\partial_{i}$ is parallel to a CKV (resp. KV), we can assume without loss of generality that $\partial_{i}$ is a $\mathrm{CKV}$ (resp. KV), by rescaling the corresponding coordinate.

Theorem 4. Let $\mathbf{E}_{i}$ be a common eigenvector of a KS-algebra $\mathcal{K}_{n}$. Then, (i) for $n>2 \mathbf{E}_{i}$ is proportional to a conformal Killing vector if and only if the ratios $f_{i}^{j h}$ are constant or undefined for every $h<j<n$; in particular, (ii) for any $n \mathbf{E}_{i}$ is proportional to a Killing vector if and only if the ratios $f_{i}^{j h}$ are constant or undefined for every $h<j$.

Proof. Let $\mathbf{E}_{i}$ be a common eigenvector of $\mathcal{K}_{n}$ and $\left(q^{i}\right)$ be separable coordinates adapted to $\mathcal{K}_{n}$. For simplicity we take $i=1$. Since the separable coordinates are orthogonal, $\mathbf{E}_{1}$ is proportional to $\partial_{1}$. Thus, without loss of generality, we assume that the vector $\mathbf{E}_{1}=\partial_{1}$ is proportional to a CKV.

From Proposition 2 we have for all $j \neq 1$

$$
\partial_{1} f_{j}^{h k}=0 .
$$

Let us consider $\partial_{1} f_{1}^{h k}$. Due to properties of determinants, we have

$$
\partial_{1} \operatorname{det} \Lambda_{k}^{1}=\sum_{p=2}^{n} \Xi_{p}
$$

where $\Xi_{p}$ is the determinant of the matrix obtained from $\Lambda_{k}^{1}$ by replacing the elements $\left(\lambda_{h}^{p}\right)$ of its $p$-th column with $\left(\partial_{1} \lambda_{h}^{p}\right)$. By equations $(2.5)_{1}$ and (4.5), for all $h, k$, we have

$$
\partial_{1} \lambda_{k}^{h}=\left(\lambda_{k}^{1}-\lambda_{k}^{h}\right) F
$$

By substituting (4.8) in (4.7), we obtain

$$
\partial_{1} \operatorname{det} \Lambda_{k}^{1}=-F\left((n-2) \operatorname{det} \Lambda_{k}^{1}-\sum_{p=1}^{n}(-1)^{p} \operatorname{det} \Lambda_{k}^{p}\right) .
$$

We remark that $\sum_{p=1}^{n}(-1)^{p} \operatorname{det} \Lambda_{k}^{p}$ is (up to the sign) the determinant of the matrix obtained from $\Lambda$ by replacing the $k$-th row with the row made by $n$ elements equal to 1 . Moreover, since for $k \neq n$ also the last row of $\Lambda_{k}^{1}$ contains $n$ elements equal to 1 , we have

$$
\sum_{p=1}^{n}(-1)^{p} \operatorname{det} \Lambda_{k}^{p}=0, \quad k \neq n, \quad \sum_{p=1}^{n}(-1)^{p} \operatorname{det} \Lambda_{n}^{p}=(-1)^{n+1} \operatorname{det} \Lambda .
$$

We can now evaluate $\partial_{1} f_{1}^{h k}$ for all indices $j, h$ such that the function is well-defined. Recalling that we always assume $k<h$, from (4.9) and (4.10) it follows

$$
\partial_{1} f_{1}^{h k}=0, \quad h \neq n, \quad \partial_{1} f_{1}^{n k}=(-1)^{n+1} F \frac{\operatorname{det} \Lambda}{\operatorname{det} \Lambda_{k}^{1}} .
$$

This proves that $f_{1}^{h k}$ is constant for $k<h<n$. In particular if $F=0$ (i.e., $\partial_{1}$ is proportional to a Killing vector), then all $f_{1}^{h k}$ are constant or undefined.

Conversely, let us assume that $f_{1}^{h k}$ is constant or undefined for every $k<h<n$. By (4.3) there exists an index $j \neq n$ and $n-1$ real constant $c^{h j}(h=1, \ldots, n-1)$ such that for all $h<n$

$$
\varphi_{1}^{(h)}=c^{h j} \varphi_{1}^{(j)}
$$


and, due to the regularity of $\mathbf{S}, \varphi_{1}^{(j)} \neq 0$. Let $\tilde{\mathbf{S}}$ be the $n \times n$ matrix obtained from $\mathbf{S}$ by dividing the first row by $\varphi_{1}^{(j)}$. The following relations link $\mathbf{S}$ and $\tilde{\mathbf{S}}$

$$
\operatorname{det} \mathbf{S}=\varphi_{1}^{(j)} \operatorname{det} \tilde{\mathbf{S}}, \quad \operatorname{det} \mathbf{S}_{h}^{k}=\varphi_{1}^{(j)} \operatorname{det} \tilde{\mathbf{S}}_{h}^{k}, \quad h \neq 1, \quad \operatorname{det} \mathbf{S}_{1}^{k}=\operatorname{det} \tilde{\mathbf{S}}_{1}^{k}
$$

The $n$-th element of the first row of $\tilde{\mathbf{S}}$ is the only element of the matrix depending on $q^{1}$. Thus,

$$
\partial_{1} \operatorname{det} \tilde{\mathbf{S}}=(-1)^{n+1} \partial_{1}\left(\frac{\varphi_{1}^{(n)}}{\varphi_{1}^{(j)}}\right) \operatorname{det} \tilde{\mathbf{S}}_{1}^{n},
$$

and for the same reason we get, up to the sign,

$$
\begin{array}{ll}
\partial_{1} \operatorname{det} \tilde{\mathbf{S}}_{h}^{k}=\partial_{1}\left(\frac{\varphi_{1}^{(n)}}{\varphi_{1}^{(j)}}\right) \operatorname{det}\left(\tilde{\mathbf{S}}_{h}^{k}\right)_{1}^{n}, & h \neq 1 \text { and } k \neq n, \\
\partial_{1} \operatorname{det} \tilde{\mathbf{S}}_{h}^{k}=0, & h=1 \text { or } k=n .
\end{array}
$$

From the definition of inverse matrix, we have

$$
\begin{aligned}
& g^{11}=\varphi_{(n)}^{1}=(-1)^{1+n} \frac{\operatorname{det} \mathbf{S}_{1}^{n}}{\operatorname{det} \mathbf{S}}=(-1)^{1+n} \frac{\operatorname{det} \tilde{\mathbf{S}}_{1}^{n}}{\varphi_{1}^{(j)} \operatorname{det} \tilde{\mathbf{S}}}, \\
& g^{h h}=\varphi_{(n)}^{h}=(-1)^{h+n} \frac{\operatorname{det} \mathbf{S}_{h}^{n}}{\operatorname{det} \mathbf{S}}=(-1)^{h+n} \frac{\operatorname{det} \tilde{\mathbf{S}}_{h}^{n}}{\operatorname{det} \tilde{\mathbf{S}}}, \quad h \neq 1 .
\end{aligned}
$$

Thus, we get

$$
\partial_{1} g^{11}=(-1)^{1+n} \partial_{1}\left(\frac{\operatorname{det} \tilde{\mathbf{S}}_{1}^{n}}{\varphi_{1}^{(j)} \operatorname{det} \tilde{\mathbf{S}}}\right), \quad \partial_{1} g^{h h}=(-1)^{h+n} \partial_{1}\left(\frac{\operatorname{det} \tilde{\mathbf{S}}_{h}^{n}}{\operatorname{det} \tilde{\mathbf{S}}}\right)
$$

for $h \neq 1$. Hence, by $(4.14)_{2}$ we have

$$
\partial_{1} g^{11}=(-1)^{n} \frac{\left(\operatorname{det} \tilde{\mathbf{S}}_{1}^{n}\right) \partial_{1}\left(\varphi_{1}^{(j)} \operatorname{det} \tilde{\mathbf{S}}\right)}{\left(\varphi_{1}^{(j)} \operatorname{det} \tilde{\mathbf{S}}\right)^{2}}, \quad \partial_{1} g^{h h}=(-1)^{h+n-1} \frac{\left(\operatorname{det} \tilde{\mathbf{S}}_{h}^{n}\right) \partial_{1}(\operatorname{det} \tilde{\mathbf{S}})}{(\operatorname{det} \tilde{\mathbf{S}})^{2}},
$$

and

$$
\partial_{1} \ln g^{h h}=-\partial_{1} \ln (\operatorname{det} \tilde{\mathbf{S}}), \quad \partial_{1} \ln g^{11}=-\partial_{1} \ln (\operatorname{det} \tilde{\mathbf{S}})-\partial_{1} \ln \varphi_{1}^{(j)} .
$$

By Lemma 1 it follows that $\partial_{1}$ is proportional to a CKV with $F=-\partial_{1} \ln (\operatorname{det} \tilde{\mathbf{S}})$ and $f=\sqrt{\varphi_{1}^{j}}$. We remark that $F$ does not depend on the choice of the element $\varphi_{1}^{(j)}$ used in the construction of $\tilde{\mathbf{S}}$. Indeed, for any other $j^{\prime}$ such that $\varphi_{1}^{\left(j^{\prime}\right)} \neq 0$, by $(4.12)$ we have

$$
\varphi_{1}^{\left(j^{\prime}\right)}=c^{j^{\prime} j} \varphi_{1}^{(j)}
$$

for a suitable constant $c^{j^{\prime} j} \in \mathbb{R}$ and $\operatorname{det} \tilde{\mathbf{S}}=c^{j^{\prime} j} \operatorname{det} \tilde{\mathbf{S}}^{\prime}$, where $\tilde{\mathbf{S}}^{\prime}$ is obtained from $\mathbf{S}$ by dividing the first row by $\varphi_{1}^{\left(j^{\prime}\right)}$. Then, the function $F$ does not change. In the particular case when all $f_{1}^{j h}$ are constant, then (4.12) hold for all $h=1, \ldots, n$ and by (4.13) it follows that $F=-\partial_{1} \ln (\operatorname{det} \tilde{\mathbf{S}})=0$. Hence, by (4.15) we get $\partial_{1} g^{h h}=0$ for any $h \neq 1$ and $\partial_{1} g^{11}=-\partial_{1} \ln \varphi_{1}^{(j)}$. According to Lemma 1 and Remark 6, this means that $\partial_{1}$ is proportional to a Killing vector and $q^{1}$ is ignorable up to a rescaling. In this case, due to $(2.5)_{1}$ all the elements of $\mathbf{S}^{-1}$ and $\mathbf{S}$ do not depend on $q^{1}$. 
Remark 7. For $n=2$ every eigenvector of a characteristic Killing tensor is proportional to a CKV. This fact can be checked directly by writing $g^{11}$ and $g^{22}$ in terms of a two dimensional Stäckel matrix.

From Proposition 2 and Theorem 4 it follows that

Theorem 5. Let $\mathbf{E}_{i}$ be a common eigenvector of a $K S$-algebra. For every $i=1, \ldots, n$ one and only one of the following statements holds: I) $\mathbf{E}_{i}$ is, up to a scalar factor, a $K V$. II) There exist indices $j, h$ such that, in a neighborhood of any point $P$ where $d f_{i}^{j h}(P) \neq 0$, the equation

$$
f_{i}^{j h}=\mathrm{const}
$$

defines a hypersurface orthogonal to $\mathbf{E}_{i}$.

Example 2. Let us consider a L-tensor $\mathbf{L}$, that is a conformal Killing tensor with simple eigenvalues and vanishing Nijenhuis torsion [5]. It is known that, if the eigenvalues $\left(u^{i}\right)$ of the L-tensor $\mathbf{L}$ are functionally independent (this property is not required in our definition according to [5]), then the $\left(u^{i}\right)$ are a separable coordinate system for the geodesic HamiltonJacobi equation. In [15] a computer algorithm was implemented for constructing of the separable coordinates associated with a L-tensor compatible with the potential of a natural Hamiltonian on a proper Riemannian manifold. We verify the behaviour of the $f_{i}^{j h}$ for the KS-algebra generated by $\mathbf{L}$. Moreover, we see that our method allows to find new properties of these systems. We recall that a symmetric two-tensor field $\mathbf{K}$ is said to be a conformal Killing tensor (CKT) if there exists a vector field $\mathbf{C}$ such that

$$
[\mathbf{K}, \mathbf{G}]=2 \mathbf{C} \odot \mathbf{G},
$$

where $[\cdot, \cdot]$ denotes the Lie-Schouten brackets and $\odot$ the symmetric tensor product. Then, (see $[5,3])$, the tensors $\left(\mathbf{K}_{0}, \mathbf{K}_{1}, \ldots, \mathbf{K}_{n-1}\right)$ where

$$
\mathbf{K}_{0}=\mathbf{G}, \quad \mathbf{K}_{a}=\frac{1}{a} \operatorname{tr}\left(\mathbf{K}_{a-1} \mathbf{L}\right) \mathbf{G}-\mathbf{K}_{a-1} \mathbf{L}, \quad a>1
$$

form a basis of a KS-algebra. The matrix $\Lambda$ of eigenvalues of the $\mathbf{K}_{a}$ is $\Lambda=\left(\sigma_{a}^{i}\left(u^{1}, \ldots, u^{n}\right)\right), a=$ $0, \ldots, n-1, i=1, \ldots, n$, where $\underline{u}=\left(u^{1}, \ldots, u^{n}\right)$ are the eigenvalues of $\mathbf{L}$ and, for $a>0, \sigma_{a}^{i}$ are the elementary symmetric polynomials of degree $a$ in the $n-1$ variables $\left(u^{1}, \ldots, u^{i-1}, u^{i+1}, \ldots, u^{n}\right)$, for $a=0$ we set $\sigma_{0}^{i}=1$. In this case we have

$$
f_{i}^{j h}=\left(u^{i}\right)^{h-j} .
$$

Indeed, we have that the inverse matrix of $\Lambda$ is

$$
\mathbf{A}=\left(A_{i}^{a}\right)=\left((-1)^{a} \frac{\left(u^{i}\right)^{n-a-1}}{U^{\prime}\left(u^{i}\right)}\right),
$$

where $U^{\prime}\left(u^{i}\right)$ is a suitable function of $u^{i}$ (see [3]) and the fundamental functions satisfy

$$
f_{i}^{j h}=\frac{\operatorname{det} \Lambda_{j}^{i}}{\operatorname{det} \Lambda_{h}^{i}}=(-1)^{j-h} \frac{A_{i}^{j}}{A_{i}^{h}} .
$$

In particular, as expected, we get $f_{i}^{j+1}=u^{i}$. We notice that, due to (4.19), for any fixed $i$ either the fundamental functions are constant for all $j<h$ (if $u^{i} \in \mathbb{R}$ ), either none of them is constant (if $u^{i}$ is a non-constant function). Thus, by Theorem 4, we obtain the following theorem, which provides an interesting restriction upon L-tensors.

Theorem 6. For $n>2$, a L-tensor has no eigenvector proportional to a proper conformal Killing vector. 


\section{Separable webs in Riemannian and pseudo-Riemannian manifolds}

In this section we prove that the method shown in Section 3 is effective also when separable coordinates are not orthogonal or the metric is not positive definite and isotropic coordinates may be present. However, for adapting the results of the orthogonal case, we have to take in account several differences occurring in the general case. First of all we recall that in the general case we cannot identify $\mathbf{E}_{i}$ and $\partial_{i}$ as seen in the previous section. Indeed, for orthogonal separable coordinates the common eigenvectors of $\mathcal{K}_{n}$ are always in the form $\mathbf{E}_{i}=f^{i} \partial_{i}$ ( $i$ not summed) as well as the corresponding eigenforms $\left(\mathbf{E}_{i}\right)^{b}=f_{i} d q^{i}$ are proportional to $d q^{i}$ (see Definition 1). In the non-orthogonal case, from Definition 1 still $d q^{a}=\left(\mathbf{E}_{a}\right)^{b}$, but by $(2.1)$

$$
\mathbf{E}_{\hat{a}}=g^{\hat{a} \hat{a}} \partial_{\hat{a}}, \quad \mathbf{E}_{\bar{a}}=g^{\bar{a} \alpha} \partial_{\alpha}=\varphi_{(m)}^{\bar{a}}\left(\theta_{\bar{a}}^{\alpha} \partial_{\alpha}\right) .
$$

Thus, for all indices $\hat{a}$ the eigenvectors $\mathbf{E}_{\hat{a}}$ are proportional to $\partial_{\hat{a}}$, but for isotropic coordinates the fields $\mathbf{E}_{\bar{a}}$ are not proportional to $\partial_{\bar{a}}$. Moreover, by $(5.1)_{2}$, we see that $\mathbf{E}_{\bar{a}}$ are proportional to the vectors $\theta_{\bar{a}}^{\alpha} \partial_{\alpha}$ which are Killing vectors of the hypersurfaces orthogonal to $\mathbf{E}_{\bar{a}}$. If for all $\alpha$ the functions $\theta_{\bar{a}}^{\alpha}$ are constant, the vectors $\theta_{\bar{a}}^{\alpha} \partial_{\alpha}$ are $\mathrm{KV}$ of the whole manifold. Since $\mathbf{E}_{\bar{a}}$ are null vectors, we can distinguish between essential eigenvectors of type $\hat{a}$ and $\bar{a}$ before knowing the separable coordinates. Moreover, in non-orthogonal coordinates the characterization of the vector fields $\partial_{a}$ associated with essential coordinates $q^{a}$ as $\mathrm{CKV}$ or $\mathrm{KV}$ is more complicated than Lemma 1:

Lemma 2. Let $\left(g^{i j}\right)$ in standard form (2.1) in coordinates $\left(q^{i}\right)=\left(q^{a}, q^{\alpha}\right)$. The vector $\mathbf{X}=\partial_{a}$ is a $C K V$ if and only if there exists a function $F$ such that

$$
\begin{aligned}
& \partial_{a} \ln g^{\hat{b} \hat{b}}=F, \\
& \partial_{a} g^{\bar{b} \alpha}=F g^{\bar{b} \alpha}, \quad \hat{b}=1, \ldots, m_{1}, \quad \bar{b}=m_{1}+1, \ldots, m . \\
& \partial_{a} g^{\alpha \beta}=F g^{\alpha \beta} .
\end{aligned}
$$

If $F=0$ then $\mathbf{X}$ is a $K V$.

Proof. From definition of CKV we have, for $\mathbf{X}=\partial_{a}$

$$
\partial_{a} g^{i j}=F g^{i j},
$$

for all $i, j=1, \ldots, n$. By (2.1), observing that $g^{\hat{b} \hat{b}} \neq 0$, we obtain (5.2).

Unlike the orthogonal case, by (2.2) and (5.2) we can see that not only the components of the inverse of a Stäckel matrix are involved in the definition of CKV, but also the functions $\theta_{\bar{a}}^{\alpha}$ and $\eta_{a}^{\alpha \beta}$. Since these functions appear in the metric components, we have to modify Theorem 4 for the non-orthogonal case. In particular we need to define a new kind of vector fields playing the role of $\mathrm{KV}$ and $\mathrm{CKV}$ in non-orthogonal separable coordinates.

Definition 4. Let $\left(q^{i}\right)=\left(q^{a}, q^{\alpha}\right)$ be standard separable coordinates. The vector $\mathbf{X}=\partial_{a}$ is a conformal Stäckel symmetry (CS-symmetry) of the foliation $S^{a}$ if there exists a function $F$ such that for all $b=1, \ldots, m$

$$
\partial_{a} \ln \varphi_{(m)}^{b}=F
$$

We say that $\mathbf{X}$ is a Stäckel symmetry (S-symmetry) if it is a CS-symmetry with $F=0$.

Remark 8. Due to the regularity of $\left(g^{i j}\right)$ the $\varphi_{(m)}^{b}$ are all different from zero. For the same reason, for a given $\bar{b}$, the $\theta_{\bar{b}}^{\alpha}$ are not all zero. 
Proposition 4. Let $\partial_{a}$ be a CS-symmetry (S-symmetry). Then, $\partial_{a}$ is a $C K V$ (KV) if and only if $\theta_{a}^{\alpha}$ and $\eta_{a}^{\alpha \beta}$ are constant for every $\alpha, \beta$.

Proof. By (2.2) and (5.3), for a given CS-symmetry $\partial_{a}$ we have

$$
\begin{aligned}
& \partial_{a} \ln g^{\hat{b} \hat{b}}=F \\
& \partial_{a} g^{\bar{b} \alpha}=\delta_{a}^{\bar{b}} \partial_{a} \theta_{\bar{b}}^{\alpha} \varphi_{(m)}^{\bar{b}}+F g^{\bar{b} \alpha}, \\
& \partial_{a} g^{\alpha \beta}=\partial_{a} \eta_{a}^{\alpha \beta} \varphi_{(m)}^{a}+F g^{\alpha \beta} .
\end{aligned}
$$

Because of (5.2) and of $\varphi_{(m)}^{a} \neq 0, \partial_{a}$ is a CKV if and only if $\theta_{\bar{a}}^{\alpha}$ and $\eta_{a}^{\alpha \beta}$, both functions of the coordinate corresponding to the lower index only, are constant for every $\alpha, \beta$.

Remark 9. Proposition 4 shows that CS-symmetries are not coordinate independent objects unless coordinates are orthogonal, in this case they coincide with CKV. Also for the isotropic coordinates we have that if $\partial_{\bar{a}}$ is a KV then, by Proposition 4 and $(5.1)_{2}, \mathbf{E}_{\bar{a}}$ is proportional to the $\mathrm{KV} \theta_{\bar{a}}^{\alpha} \partial_{\alpha}$. If no isotropic coordinate occurs, then $\partial_{a}$ is a CS-symmetry if and only if it is a CKV of each $m$-dimensional submanifold $\left\{q^{\alpha}=\right.$ const, $\left.\alpha=m+1, \ldots, n\right\}$.

As in the previous sections, we introduce the $m \times m$ matrix $\boldsymbol{\Lambda}=\left(\lambda_{b}^{a}\right)$ of the essential eigenvalues of a basis $\left(\mathbf{K}_{1}, \ldots, \mathbf{K}_{m}=\mathbf{G}\right)$ of $\mathcal{K}_{m}$ described in Section 3. Even if in the construction of $\boldsymbol{\Lambda}$ we do not explicitly distinguish between eigenvalues $\lambda^{\hat{a}}$ and $\lambda^{\bar{a}}$, we will see in Remark 13 that the distinction is relevant.

Proposition 5. The vector $\mathbf{X}=\partial_{a}$ is a CS-symmetry if and only if

$$
\partial_{a} \lambda_{c}^{b}=\left(\lambda_{c}^{a}-\lambda_{c}^{b}\right) F \quad \forall b, c=1, \ldots, m,
$$

where $F$ is the function appearing in (5.3). The vector $\mathbf{X}=\partial_{a}$ is a S-symmetry if and only if

$$
\partial_{a} \lambda_{c}^{b}=0 \quad \forall b, c=1, \ldots, m
$$

Proof. It follows directly from Definition 4 and $(2.5)_{1}$, recalling that in $\left(\mathcal{K}_{m}\right)$ there is always at least one KT with distinct essential eigenvalues.

Lemma 3. Let $S=\left(\varphi_{a}^{(b)}\right)$ be the $m \times m$ Stäckel matrix defined by (2.2) and (2.4). Then

$$
\varphi_{(b)}^{a}=\lambda_{b}^{a} \varphi_{(m)}^{a}, \quad \forall a, b=1, \ldots, m .
$$

Proof. According to (2.3) and (2.4), the non-vanishing components of the basis $\left(\mathbf{K}_{1}, \ldots, \mathbf{K}_{m}\right)$ in standard coordinates have the following equivalent forms

$$
K_{b}^{\hat{a} \hat{a}}=\lambda_{b}^{\hat{a}} g^{\hat{a} \hat{a}}=\varphi_{(b)}^{\hat{a}}, \quad K_{b}^{\bar{a} \beta}=\lambda_{b}^{\bar{a}} g^{\bar{a} \beta}=\theta_{\bar{a}}^{\beta} \varphi_{(b)}^{\bar{a}} .
$$

By inserting the expression of the metric (2.2) in (5.6), we get

$$
\lambda_{b}^{\hat{a}} \varphi_{(m)}^{\hat{a}}=\varphi_{(b)}^{\hat{a}}, \quad \lambda_{b}^{\bar{a}} \theta_{\bar{a}}^{\beta} \varphi_{(m)}^{\bar{a}}=\theta_{\bar{a}}^{\beta} \varphi_{(b)}^{\bar{a}} .
$$

Hence, due to Remark 8, relation (5.5) holds for all essential eigenvalues, without distinction between isotropic and non-isotropic coordinates.

Remark 10. By (5.5) for the general separation (cf. (4.4) for the orthogonal case), it follows: $\operatorname{det} \mathbf{S}^{-1}=\operatorname{det} \Lambda \prod_{a} \varphi_{(m)}^{a}$. Therefore, for nondegenerate metrics we have always $\operatorname{det} \Lambda \neq 0$. 
We define for essential coordinates the fundamental functions $f_{a}^{b c}$ in terms of minors of $\Lambda$ as described by (3.1). Namely, Propositions 2 and 3 can be directly restated here as follows

Proposition 6. (i) If

$$
f_{a}^{b c}=\frac{\operatorname{det} \Lambda_{b}^{a}}{\operatorname{det} \Lambda_{c}^{a}}=(-1)^{b+c} \frac{\varphi_{a}^{(b)}}{\varphi_{a}^{(c)}}
$$

is well-defined, then it depends on $q^{a}$ only. (ii) For any fixed index a there exist two indices $c<b \leq m$ such that the fundamental function $f_{a}^{b c}(5.7)$ is well-defined.

We can now generalize Theorem 4:

Theorem 7. Let $q^{a}$ be an essential coordinate adapted to a separable Killing algebra $\left(D_{r}, \mathcal{K}_{m}\right)$. Then, (i) for $m>2$ there exists a rescaling $\breve{q}^{a}=\breve{q}^{a}\left(q^{a}\right)$ such that the associated vector field $\breve{\partial}_{a}$ is a CS-symmetry if and only if the functions $f_{a}^{b c}$ (5.7) are constant or undefined for every $c<b<m$. In particular, (ii) for any $m, \breve{\partial}_{a}$ is a S-symmetry if and only if the functions $f_{a}^{b c}$ are constant or undefined for every indices $b, c$.

Proof. By comparing (5.5) and (4.1), we see that the relations between essential components of Killing tensors and the $m \times m$ Stäckel matrix $\left(\varphi_{c}^{(a)}\right)$ are exactly the same as in the orthogonal case. To prove our thesis, we follow the proof of Theorem 4 with some modifications. Let us assume $a=1$ and that $\breve{\partial}_{1}$ is a CS-symmetry. Then, equations (5.4) hold and by calculating $\breve{\partial}_{1} f_{1}^{b c}$ as in Theorem 4 we get equations

$$
\breve{\partial}_{1} f_{1}^{b c}=0, \quad c<b<m, \quad \breve{\partial}_{1} f_{1}^{m c}=(-1)^{m+1} F \frac{\operatorname{det} \Lambda}{\operatorname{det} \Lambda_{c}^{1}},
$$

corresponding to (4.11). Hence, the fundamental functions $f_{1}^{b c}$ are constant or undefined for all $b<c<m$ and, if $F=0$ (i.e., $\breve{\partial}_{1}$ is a S-symmetry) they are all constant or undefined. Conversely, if $f_{1}^{b c}$ are constant or undefined for all $b<c<m$, by repeating the same reasoning of Theorem 4 we obtain the following equations analogous to (4.16)

$$
\partial_{1} \ln \varphi_{(m)}^{b}=-\partial_{1} \ln (\operatorname{det} \tilde{\mathbf{S}}), \quad \partial_{1} \ln \varphi_{(m)}^{1}=-\partial_{1} \ln (\operatorname{det} \tilde{\mathbf{S}})-\partial_{1} \ln \varphi_{1}^{(c)},
$$

with $b \neq 1$, where $\varphi_{1}^{(c)}$ is a non-vanishing element of the first row of the Stäckel matrix $\mathbf{S}$ and $\tilde{\mathbf{S}}$ is the matrix obtained from $\mathbf{S}$ by dividing the first row by $\varphi_{1}^{(c)}$. If $\partial_{1} \varphi_{1}^{(c)} \neq 0$, we can locally rescale $q^{1}$ as $\breve{q}^{1}=\varphi_{1}^{(c)}\left(q^{1}\right.$ ) (if $\varphi_{1}^{(c)}$ is constant we do not need to rescale and $\breve{\partial}_{1}=\partial_{1}$ ). Hence, for all $b=1, \ldots, m$ we get $\breve{\partial}_{1} \ln \varphi_{(m)}^{b}=-\breve{\partial}_{1} \ln (\operatorname{det} \tilde{\mathbf{S}})$ and by (5.3) $\breve{\partial}_{1}$ is a CS-symmetry with $F=-\breve{\partial}_{1} \ln (\operatorname{det} \tilde{\mathbf{S}})$. In particular, as in the orthogonal case, if all $f_{1}^{b c}$ are constant or undefined then $\operatorname{det} \tilde{\mathbf{S}}$ is independent of $\breve{q}^{1}, F=0$, and $\breve{\partial}_{1}$ is a S-symmetry.

Remark 11. In the previous theorem no distinction is made between coordinates of type $\hat{a}$ and $\bar{a}$. For $m=2$ it is easy to check that every $\partial_{a}$ is up to a rescaling a CS-symmetry.

By Theorem 7, Theorem 5 can be generalized in the following way

Theorem 8. Let $\left(q^{a}\right)$ be essential coordinates adapted to a separable Killing algebra $\left(D_{r}, \mathcal{K}_{m}\right)$. For every $a=1, \ldots, m$ one and only one of the following statements holds: I) there exists a rescaling $\breve{q}^{a}=\breve{q}^{a}\left(q^{a}\right)$ such that the associated vector field $\breve{\partial}_{a}$ is a S-symmetry. II) There exist indices $b, c$ such that, in a neighborhood of any point where $d f_{a}^{b c} \neq 0$, the equation

$$
f_{a}^{b c}=\text { const }
$$

defines a hypersurface of the foliation $q^{a}=$ const. 
Remark 12. The vector field $\partial_{a}$ is a S-symmetry if and only if the Stäckel matrix does not depend on $q^{a}$. Therefore, unlike the orthogonal case, item I) of Theorem 8 does not provide a geometric characterization of the field $\partial_{a}$, but merely a property of the Stäckel matrix with respect to $\left(q^{i}\right)$, as it is illustrated in Example 3. On the contrary, item II) retains the same geometric meaning as in Theorem 5 .

Remark 13. Since $\mathbf{E}_{\hat{a}}$ is proportional to $\partial_{\hat{a}}$, by applying Theorems 7 and 8 to the fundamental functions $f_{\hat{a}}^{b c}$ we have that if $\partial_{\hat{a}}$ is a CS-symmetry then $\partial_{\hat{a}}$ is a common eigenvector of $\mathcal{K}_{m}$ orthogonal to $S^{\hat{a}}$. This is not true for indices $\bar{a}$, which correspond to isotropic eigenvectors $\mathbf{E}_{\bar{a}}$ generating the isotropic distribution $I=\Delta \cap \Delta^{\perp}$.

Example 3. Let us consider the Euclidean four-dimensional space $\mathbb{R}_{4}$. Let $\mathcal{S}_{2}$ be the set of two foliations $S^{1}$ and $S^{2}$ described in Cartesian coordinates $(x, y, z, t)$ as

$$
S^{1}=\bigcup_{k>0}\left\{(x, y, z, t) \in \mathbb{R}^{4} \mid x^{2}+y^{2}=k\right\}, \quad S^{2}=\bigcup_{h \in \mathbb{R}}\left\{(x, y, z, t) \in \mathbb{R}^{4} \mid t=h\right\} .
$$

Two vectors orthogonal to $S^{1}$ and $S^{2}$ respectively are $\mathbf{n}_{1}=x \partial_{x}+y \partial_{y}, \mathbf{n}_{2}=\partial_{t}$. Let $D_{2}$ be the linear space generated by the vectors

$$
\mathbf{X}_{3}=\partial_{z}, \quad \mathbf{X}_{4}=y \partial_{x}-x \partial_{y}
$$

which are commuting Killing vectors tangent to both foliations $S^{a}$. The tensor

$$
\mathbf{K}=\partial_{t} \otimes \partial_{t}
$$

is a $D_{2}$-invariant KT. Moreover, $\mathbf{E}_{1}=\mathbf{n}_{1}$ and $\mathbf{E}_{2}=\mathbf{n}_{2}$ are eigenvectors of $\mathbf{K}$ associated with the eigenvalues $\lambda^{1}=0$ and $\lambda^{2}=1$ respectively. Hence, according to Definition $2,\left(\mathcal{S}_{2}, D_{2}, \mathbf{K}\right)$ is a separable Killing web. The tensors $(\mathbf{K}, \mathbf{G})$ form a basis of the KT-space $\mathcal{K}_{2}$. Let us construct the adapted coordinates $\left(q^{a}, q^{\alpha}\right)$ and compute the components of the metric in these new coordinates. As essential coordinates we choose $q^{1}=\sqrt{x^{2}+y^{2}}=\rho$ and $q^{2}=t$. By adding the ignorable coordinates $\left(q^{\alpha}\right)=\left(q^{3}, q^{4}\right)$ associated with the basis $\left(\mathbf{X}_{3}, \mathbf{X}_{4}\right)$ and with a section $\mathcal{Z}$ orthogonal to the orbits of $D_{2}$, the coordinate transformation is defined by

$$
x=\rho \cos \left(q^{4}+\theta_{0}\right), \quad y=\rho \sin \left(q^{4}+\theta_{0}\right), \quad z=q^{3}+z_{0}, \quad t=q^{2},
$$

where $\theta_{0} \in(0,2 \pi)$ and $z_{0} \in \mathbb{R}$ are the parameters defining $\mathcal{Z}$. In these coordinates the metric is diagonal and the non vanishing components of $\mathbf{G}$ are

$$
g^{11}=g^{22}=g^{44}=1, \quad g^{33}=\rho^{-2} .
$$

By choosing a different basis of $D_{2}$, for instance $\mathbf{X}_{3}^{\prime}=\mathbf{X}_{3}$ and $\mathbf{X}_{4}^{\prime}=\mathbf{X}_{3}+\mathbf{X}_{4}$, and leaving $\mathcal{Z}$ unchanged, we get non-orthogonal ignorable coordinates $\left(q^{\prime \alpha}\right)$ given by

$$
x=\rho \cos \left(q^{\prime 4}+\theta_{0}\right), \quad y=\rho \sin \left(q^{4}+\theta_{0}\right), \quad z=q^{\prime 3}+q^{\prime 4}+z_{0}, \quad t=q^{2} .
$$

and the metric assumes the standard form

$$
\mathbf{G}=\left(\begin{array}{cccc}
1 & 0 & 0 & 0 \\
0 & 1 & 0 & 0 \\
0 & 0 & 1+\rho^{-2} & -1 \\
0 & 0 & -1 & 1
\end{array}\right)
$$

In both cases the $2 \times 2$ Stäckel matrix associated with the essential separable coordinates and its inverse are

$$
\mathbf{S}=\left(\begin{array}{cc}
-1 & 1 \\
1 & 0
\end{array}\right), \quad \mathbf{S}^{-1}=\left(\begin{array}{cc}
\lambda^{1} g^{11} & \lambda^{2} g^{22} \\
g^{11} & g^{22}
\end{array}\right)=\left(\begin{array}{ll}
0 & 1 \\
1 & 1
\end{array}\right)
$$


respectively. The matrix $\Lambda$ of the essential eigenvalues of $\mathbf{K}$ and $\mathbf{G}$ coincides with the mat$\operatorname{rix} \mathbf{S}^{-1}$. The method of the eigenvalues does not provide any coordinate hypersurface because $\mathbf{S}$ is constant. Then, according to Theorem 8, $\partial_{1}, \partial_{2}$ are both S-symmetries. However, from a geometric point of view the corresponding eigenvectors $\mathbf{E}_{1}=x \partial_{x}+y \partial_{y}$ and $\mathbf{E}_{2}=\partial_{t}$ have different properties. Indeed, $\mathbf{E}_{2}$ is a $\mathrm{KV}$, according to the fact that $q^{2}$ is ignorable, while $\mathbf{E}_{1}$ is not a $\mathrm{KV}$ since $g^{33}$ depends on $q^{1}$. We can apply the eigenvalue method to the orthogonal system (5.8) for computing the equation of the hypersurfaces of $S^{1}$. In order to determine the $4 \times 4$ matrix $\Lambda$ we consider the 4-dimensional $\mathrm{KT}$ space containing $\mathcal{K}_{2}$ and the tensors $\mathbf{K}_{2}=\mathbf{X}_{3} \otimes \mathbf{X}_{3}, \mathbf{K}_{3}=\mathbf{X}_{4} \otimes \mathbf{X}_{4}$, which is a KS-algebra for the orthogonal system (5.8).

Example 4. Let us consider a 4-dimensional pseudo-Riemannian manifold having in a coordinate system $(X, Y, Z, U)$ the following non-zero contravariant metric components

$$
\begin{aligned}
g^{11} & =-X^{10} \frac{-4\left(X^{4}-Y^{4}\right)+9 X^{4} Y^{24} \Psi}{144\left(X^{10}+Y^{10}\right)^{2}\left(X^{4}-Y^{4}\right)}, \\
g^{22} & =-Y^{10} \frac{-4\left(X^{4}-Y^{4}\right)-9 X^{24} Y^{4} \Psi}{144 X^{10}\left(X^{10}+Y^{10}\right)^{2}\left(X^{4}-Y^{4}\right)}, \\
g^{12} & =X^{5} Y^{5} \frac{4\left(X^{4}-Y^{4}\right)+9 X^{14} Y^{14} \Psi}{144\left(X^{10}+Y^{10}\right)^{2}\left(X^{4}-Y^{4}\right)}, \\
g^{33} & =-(Z-U)^{2}-\Psi, \quad g^{44}=(Z-U)^{2}-\Psi, \quad g^{34}=-\Psi,
\end{aligned}
$$

where $\Psi=\left(U-Z+X^{6}+Y^{6}\right)$. For $|Y|<|X|$ and $\Psi>0$ the signature is (3,1), while for $|Y|>|X|$ and $\Psi<0$ the signature is $(2,2)$. The 2-dimensional space of the KVs is generated by $\mathbf{X}_{1}=\partial_{Z}+\partial_{U}, \mathbf{X}_{2}=\sqrt{2|f|}\left(X^{5} \partial_{X}-Y^{5} \partial_{Y}\right)$, where

$$
f=\frac{Y^{14} X^{14}}{32\left(X^{10}+Y^{10}\right)^{2}\left(X^{4}-Y^{4}\right)} .
$$

We call $D_{1}$ the KV space generated by $\mathbf{X}_{1}$. We have $r=1=m_{0}$, since $\mathbf{X}_{1}$ is an isotropic vector. Let us consider the independent tensors $\mathbf{K}_{1}, \mathbf{K}_{2}$ whose non null contravariant components are

$$
\begin{aligned}
& K_{1}^{11}=Y^{10}(U-Z) f, \quad K_{1}^{22}=X^{10}(U-Z) f, \quad K_{1}^{12}=-X^{5}-Y^{5}(U-Z) f, \\
& K_{1}^{33}=1+\frac{1}{2}(Z-U)^{2}-\frac{1}{2}(Z-U), \quad K_{1}^{34}=1-\frac{1}{2}(Z-U), \\
& K_{1}^{44}=1-\frac{1}{2}(Z-U)^{2}-\frac{1}{2}(Z-U), \\
& K_{2}^{11}=2 Y^{10} f, \quad K_{2}^{22}=2 X^{10} f, \quad K_{2}^{12}=-2 X^{5} Y^{5} f, \quad K_{2}^{33}=K_{2}^{34}=K_{2}^{44}=1 .
\end{aligned}
$$

The space $\mathcal{K}_{3}$ generated by $\left(\mathbf{K}_{1}, \mathbf{K}_{2}, \mathbf{G}\right)$ satisfies the hypotheses of Theorem 2 . Thus, we can apply the eigenvalue method to get the equations of the separated coordinate hypersurfaces. The matrix of the eigenvalues is

$$
\Lambda=\left(\begin{array}{ccc}
0 & \frac{Z-U}{2 \Psi} & -\frac{1}{2} \\
0 & -\frac{1}{\Psi} & 0 \\
1 & 1 & 1
\end{array}\right)
$$

We get

$$
f_{1}^{23}=-X^{6}-Y^{6}, \quad f_{2}^{a b} \quad \text { constant or n.d., } \quad f_{3}^{21}=\frac{1}{2}(U-Z) .
$$

This means that

$$
x=X^{6}+Y^{6}, \quad z=(Z-U) / 2
$$


are essential separable coordinates (the last one is a null coordinate) and that, up to a rescaling, the vector $\partial_{y}$ associated with the remaining essential separable coordinate $y$ is a S-symmetry. The separable coordinate $y=1 / Y^{4}-1 / X^{4}$ cannot be computed by the eigenvalue method. The coordinate associated with $\mathbf{X}_{1} \in D_{1}$ is $u=(U+Z) / 2$. By performing the change of variables $(X, Y, Z, U) \rightarrow(x, y, z, u)$ we get the metric in standard form

$$
\mathbf{G}=\left(\begin{array}{cccc}
1 & 0 & 0 & 0 \\
0 & \frac{2 x-z}{y} & 0 & 0 \\
0 & 0 & 0 & -2 z^{2} \\
0 & 0 & -2 z^{2} & 2 z-x
\end{array}\right) .
$$

In the separable coordinates the general KV is $c_{1} \frac{1}{\sqrt{y}} \partial_{y}+c_{2} \partial_{u}$ and the tensors $\mathbf{K}_{1}, \mathbf{K}_{2}$ become

$$
\mathbf{K}_{1}=-\frac{z}{y} \partial_{y} \odot \partial_{y}+2 z^{2} \partial_{z} \odot \partial_{u}+(1-z) \partial_{u} \odot \partial_{u}, \quad \mathbf{K}_{2}=\frac{1}{y} \partial_{y} \odot \partial_{y}+\partial_{u} \odot \partial_{u} .
$$

We remark that $\mathbf{K}_{2}$ is a reducible $\mathrm{KT}$ (i.e., sum of symmetric products of $\mathrm{KV}$ ), while $\mathbf{K}_{1}$ is an irreducible tensor.

\section{Conformal separable orthogonal systems.}

The method developed in Section 3 characterizes also conformal separable orthogonal webs [7] in a natural way. We recall that

Definition 5. The geodesic Hamiltonian $G$ is conformal separable if there exists a function $\sigma$ on $Q$ such that the conformal geodesic Hamiltonian $\bar{G}=G / \sigma$ (associated with the conformal metric $\overline{\mathbf{G}}=\mathbf{G} / \sigma)$ is separable. We call conformal separable the coordinates $\left(q^{i}\right)$ allowing the separation of $\bar{G}$.

Remark 14. An important application of conformal separable coordinates is the fact that coordinates allowing $R$-separation of the Laplace equation are necessarily conformal separable (see $[19,12])$. Moreover, in conformally flat manifolds, all the conformal separable coordinates are also $R$-separable (see [16]).

Due to Definition 5, the conformal separation in orthogonal coordinates is equivalent to the existence of a KS-algebra for a conformal metric $\overline{\mathbf{G}}$. The following theorem contains an intrinsic characterization in terms of the original metric tensor $\mathbf{G}$, involving conformal Killing tensors (CKT), introduced in Example 2.

Theorem 9 ([7]). The geodesic Hamiltonian $G$ is conformal separable in orthogonal coordinates if and only if there exist $n C K T\left(\mathbf{K}_{i}\right)$ pointwise independent with common eigenvectors $\left(\mathbf{E}_{i}\right)$ and in conformal involution (i.e., there exist vector fields $\mathbf{C}_{i j}$ such that $\left[\mathbf{K}_{i}, \mathbf{K}_{j}\right]=\mathbf{C}_{i j} \odot \mathbf{G}$ ). It is not restrictive to assume $\mathbf{K}_{n}=\mathbf{G}$. Each conformal separable coordinate hypersurface is orthogonal to one of the $n$ common normal eigenvectors of $\left(\mathbf{K}_{i}\right)$.

We have $[18,7]$

Proposition 7. Let $\left(\mathbf{K}_{1}, \ldots, \mathbf{K}_{n}=\mathbf{G}\right)$ be a set of independent CKT associated with conformal separable orthogonal coordinates $\left(q^{i}\right)$, and let $\left(\lambda_{i}^{j}\right)$ be their eigenvalues with respect to the metric $\mathbf{G}$. Then, for any choice of the index $k=1, \ldots, n$, the tensors

$$
\overline{\mathbf{K}}_{i}=\mathbf{K}_{i}-\lambda_{i}^{k} \mathbf{G} \quad i=1, \ldots, n-1
$$

are $K T$ for the metric

$$
\overline{\mathbf{K}}_{n}=\overline{\mathbf{G}}=\left(g^{k k}\right)^{-1} \mathbf{G}
$$

and $\left(\overline{\mathbf{K}}_{1}, \ldots, \overline{\mathbf{K}}_{n}=\overline{\mathbf{G}}\right)$ is a basis for the KS-algebra associated with $\left(q^{i}\right)$. 
Remark 15. We say that $\left(\mathbf{K}_{1}, \ldots, \mathbf{K}_{n-1}, \mathbf{G}\right)$ is a basis of the conformal Killing space (CKspace) associated with the conformal separable coordinates $\left(q^{i}\right)$.

Due to Proposition 7 , by considering the matrix $\bar{\Lambda}$ made by the eigenvalues of $\left(\overline{\mathbf{K}}_{i}\right)$ with respect to $\overline{\mathbf{G}}$, we can apply Theorems 4, 5 for characterizing any orthogonal conformal separable web associated with $\left(\mathbf{K}_{1}, \ldots, \mathbf{K}_{n-1}, \mathbf{G}\right)$ as orthogonal separable web associated with the KSalgebra generated by $\left(\overline{\mathbf{K}}_{1}, \ldots, \overline{\mathbf{K}}_{n-1}, \overline{\mathbf{G}}\right)$. Following Section 3, we define

$$
\bar{f}_{i}^{j h}=(-1)^{j+h} \frac{\operatorname{det} \bar{\Lambda}_{j}^{i}}{\operatorname{det} \bar{\Lambda}_{h}^{i}},
$$

where the matrix $\bar{\Lambda}$ is formed by the eigenvalues of $\left(\overline{\mathbf{K}}_{i}\right)$ with respect to $\overline{\mathbf{G}}$, and

$$
f_{i}^{j h}=(-1)^{j+h} \frac{\operatorname{det} \Lambda_{j}^{i}}{\operatorname{det} \Lambda_{h}^{i}},
$$

where $\Lambda$ is the matrix made by eigenvalues of $\left(\mathbf{K}_{i}\right)$ with respect to $\mathbf{G}$. We remark that functions (6.3) are not intrinsically defined, since to determine the $\overline{\mathbf{G}}$-eigenvalues of the tensors $\left(\overline{\mathbf{K}}_{i}\right)$ it is necessary to know the coordinates because of the factor $g^{k k}$ appearing in (6.2). On the contrary, in functions (6.4) only the eigenvalues of tensors satisfying intrinsic conditions (the hypotheses of Theorem 9) are involved.

Remark 16. Definition 5 of $\left(\overline{\mathbf{K}}_{i}\right)$ implies that in $\bar{\Lambda}$ the $k$-th column has $n-1$ zeros.

Proposition 8. Let $\bar{f}_{i}^{j h}$ and $f_{i}^{j h}$ be the functions defined in (6.3) and (6.4) respectively. Then, for $h<j<n(n>2)$ we have either

$$
\bar{f}_{i}^{j h}=f_{i}^{j h},
$$

or both functions are undefined.

Proof. The eigenvalues of $\overline{\mathbf{K}}_{i}$ with respect to $\overline{\mathbf{G}}$ are

$$
\bar{\lambda}_{i}^{j}=\left(\lambda_{i}^{j}-\lambda_{i}^{k}\right) g^{k k} .
$$

By linear algebra arguments, we have that

$$
\operatorname{det} \bar{\Lambda}_{h}^{i}=\left(g^{k k}\right)^{n-2} \operatorname{det} \Lambda_{h}^{i}
$$

Hence, by (6.3) and (6.4) the thesis follows.

Remark 17. A vector field $\mathbf{X}$ is $\mathrm{CKV}$ for $\mathbf{G}$ if and only if it is a CKV for any metric conformal to $\mathbf{G}$. Thus, in the following for CKV we shall not specify which is the metric tensor considered.

In the case of the orthogonal conformal separation Theorems 4 and 5 can be restated as follows

Theorem 10. Let $\mathbf{E}_{i}$ be a common eigenvector of a basis $\left(\mathbf{K}_{i}\right)$ of a CK-space. Then, $\mathbf{E}_{i}$ is proportional to a $C K V$ if and only if for all $h<j<n(n>2)$ the functions $f_{i}^{j h}$ defined by (6.4) are constant or undefined.

Proof. Due to Proposition 8 and Remark 17, the thesis follows by applying Theorem 4 (i) to the KS-algebra (6.1), (6.2) with $k \neq i$. 
Theorem 11. Let $\mathbf{E}_{i}$ be a common eigenvector of a basis $\left(\mathbf{K}_{i}\right)$ of a CK-space. For every $i=1, \ldots, n(n>2)$ one and only one of the following statements holds: I) $\mathbf{E}_{i}$ is, up to a scalar factor, a CKV. II) There exist indices $h<j<n$ such that, in a neighborhood of any point where $d f_{i}^{j h} \neq 0$, the equation

$$
f_{i}^{j h}=\mathrm{const}
$$

defines a hypersurface orthogonal to $\mathbf{E}_{i}$.

In the case of conformal orthogonal separation, we do not distinguish if $\mathbf{E}_{i}$ is proportional to a CKV or a KV. The following property holds

Proposition 9. If $\mathbf{E}_{i}$ is, up to a factor, a $C K V$, then it is a $K V$ of $\overline{\mathbf{G}}=\mathbf{G} / g^{k k}$ for any $k \neq i$.

Proof. By (6.1), we get a basis of the KS-algebra with respect to $\mathbf{G} / g^{k k}$ with $k \neq i$. According to Remark 16, the $k$-th column of $\bar{\Lambda}$ has $n-1$ zeros. Therefore, all submatrices of kind $\bar{\Lambda}_{n}^{h}$, $h \neq k$ have null determinants. This means that for all $h<n$ the functions $\bar{f}_{i}^{h n}$ are undefined or identically null. Moreover, since according to Remark $17 \mathbf{E}_{i}$ is up to a factor a CKV for $\overline{\mathbf{G}}=\mathbf{G} / g^{k k}$, due to Theorem 4 (i) we get that for $h<j<n$ the functions $\bar{f}_{i}^{h j}=f_{i}^{h j}$ are constant or undefined. Then, Theorem 4 (ii) implies that $\mathbf{E}_{i}$ is a $\mathrm{KV}$ for $\mathbf{\mathbf { G }}$.

Example 5. Let us consider in $\mathbb{R}^{3}$ the vector fields $\mathbf{R}_{3}$ and $\mathbf{I}_{3}$ having Cartesian components

$$
\mathbf{R}_{3}=(-y, x, 0), \quad \mathbf{I}_{3}=\left(-2 x z,-2 y z, x^{2}+y^{2}-z^{2}\right),
$$

respectively. The vector $\mathbf{I}_{3}$ is a $\mathrm{CKV}$ with respect to the Euclidean metric $\mathbf{G}$ : it is the inversion with respect to a generic point on the axis $z$. The vector $\mathbf{R}_{3}$ is the rotation around the $z$ axis and it is a Killing vector. It is straightforward to check that the two vector fields commute so that the corresponding linear first integrals are in involution. Moreover, the tensors $\left(\mathbf{K}_{1}=\right.$ $\left.\mathbf{I}_{3} \otimes \mathbf{I}_{3}, \mathbf{K}_{2}=\mathbf{R}_{3} \otimes \mathbf{R}_{3}, \mathbf{G}\right)$ are pointwise independent. Hence, they satisfy the hypotheses of Theorem 9 and they are associated with some conformal separable coordinate system. We apply the above described method to determine the conformal separable coordinate hypersurfaces. Being $\mathbf{R}_{3} \perp \mathbf{I}_{3}$, the common eigenvectors are

$$
\mathbf{E}_{1}=\mathbf{I}_{3} \times \mathbf{R}_{3}, \quad \mathbf{E}_{2}=\mathbf{I}_{3}, \quad \mathbf{E}_{3}=\mathbf{R}_{3} .
$$

The eigenvalues matrix $\Lambda$ is

$$
\Lambda=\left(\begin{array}{ccc}
0 & \mathbf{I}_{3} \cdot \mathbf{I}_{3} & 0 \\
0 & 0 & \mathbf{R}_{3} \cdot \mathbf{R}_{3} \\
1 & 1 & 1
\end{array}\right)
$$

The coordinate hypersurfaces orthogonal to $\mathbf{E}_{1}$ are the level sets of the function

$$
f_{1}^{21}=\frac{\left|\begin{array}{cc}
\mathbf{I}_{3} \cdot \mathbf{I}_{3} & 0 \\
1 & 1
\end{array}\right|}{\left|\begin{array}{cc}
0 & \mathbf{R}_{3} \cdot \mathbf{R}_{3} \\
1 & 1
\end{array}\right|}=\frac{\mathbf{I}_{3} \cdot \mathbf{I}_{3}}{-\mathbf{R}_{3} \cdot \mathbf{R}_{3}}=-\frac{\left(x^{2}+y^{2}+z^{2}\right)^{2}}{x^{2}+y^{2}}
$$

which describes the rotational surface obtained by rotating around the $z$-axis a circle in the plane $(x, z)$ tangent in the origin $O$ to the $z$-axis (toroids without center opening). Since $f_{1}^{12}$ is not constant and both the upper indices are different from 3, the eigenvector $\mathbf{E}_{1}$ is not proportional to a CKV. According to the fact that $\mathbf{I}_{3}$ and $\mathbf{R}_{3}$ are conformal Killing tensors, all functions $f_{i}^{j h}$ for $i=2,3$ and $h, j \neq 3$ are constant or undefined. It is well known that the surfaces orthogonal 
to $\mathbf{E}_{3}=\mathbf{R}_{3}$ are half-planes issued from the $z$-axis. Moreover, it is easy to check that the spheres tangent in $O$ to the $x y$-plane are hypersurfaces orthogonal to $\mathbf{E}_{2}=\mathbf{I}_{3}$. Indeed, the gradient of the function $q^{2}=\left(x^{2}+y^{2}+z^{2}\right) / z$ is up to the factor $z^{2}$ exactly $\mathbf{I}_{3}$. The coordinates associated with $\left(\mathbf{K}_{1}, \mathbf{K}_{2}, \mathbf{G}\right)$ are known as tangent-spheres coordinates [20] related to $(x, y, z)$ by

$$
x=\frac{\mu \cos \psi}{\mu^{2}+\nu^{2}}, \quad y=\frac{\mu \sin \psi}{\mu^{2}+\nu^{2}}, \quad z=\frac{\nu}{\mu^{2}+\nu^{2}},
$$

where $q^{1}=\mu, q^{2}=\nu, q^{3}=\psi$ (see also [10] for a detailed analysis and classification of the symmetric conformal separable coordinates in $\mathbb{R}^{3}$ and the associated CKTs). A conformal metric which is separable in these coordinates is for instance $\overline{\mathbf{G}}=\left(\mathbf{R}_{3} \cdot \mathbf{R}_{3}\right) \mathbf{G}$. By Proposition 7 , the tensors $\mathbf{K}_{1}$ and $\mathbf{K}_{2}$ are $\mathrm{KT}$ for $\overline{\mathbf{G}}$. By Proposition $9, \mathbf{E}_{2}$ is a $\mathrm{KV}$ for $\overline{\mathbf{G}}$. By definition of $\mathbf{K}_{2}$ and because it is a $\mathrm{KT}$ for $\overline{\mathbf{G}}, \mathbf{E}_{3}$ also is a $\mathrm{KV}$ for $\overline{\mathbf{G}}$.

\section{Conclusion}

By using simple arguments of linear algebra and the properties of the Stäckel matrices, we have seen how to construct separable hypersurfaces by means of eigenvalues of symmetric twotensors in Riemannian and pseudo-Riemannian manifolds. It follows that the webs associated with these hypersurfaces have the same domain of definition of the eigenvalues employed in the costruction, apart some closed singular set where the common eigenspaces of the tensors in the KS (CKS) spaces are not one-dimensional. In (real) pseudo-Riemannian manifolds, KTs (and CKTs) may have complex conjugated eigenvalues, in this case it is not possible to define real separable coordinates. However, it is possible to introduce separated complex variables allowing the Jacobi integration (see [13]). The application of our eigenvalue method to the complex case is in progress [14]. For manifolds of constant curvature the whole spaces of Killing and conformal-Killing tensors are well known, then it is possible to apply our method to get computer-graphical representations of the webs. We remark that the separable (resp. conformalseparable) coordinates here considered are the only ones allowing separation (resp. Fixed Energy $R$-separation [12]) of Laplace, Helmholtz and Schrödinger equations [6].

\section{Acknowledgements}

This research is partially supported by MIUR (National Research Project "Geometry of Dynamical System") and by the research project "Progetto Lagrange" of Fondazione CRT.

\section{References}

[1] Benenti S., Separability structures on Riemannian manifolds, Lecture Notes in Math. 863 (1980), 512-538.

[2] Benenti S., Separation of variables in the geodesic Hamilton-Jacobi equation, Progr. Math. 9 (1991), 1-36.

[3] Benenti S., Inertia tensors and Stäckel systems in the Euclidean spaces, Rend. Semin. Mat. Univ. Polit. Torino 50 (1992), 315-341.

[4] Benenti S., Intrinsic characterization of the variable separation in the Hamilton-Jacobi equation, J. Math. Phys. 38 (1997), 6578-6602.

[5] Benenti S., Separability in Riemannian manifolds, Phil. Trans. Roy. Soc. A, to appear.

[6] Benenti S., Chanu C., Rastelli G., Remarks on the connection between the additive separation of the Hamilton-Jacobi equation and the multiplicative separation of the Schroedinger equation. 1. The completeness and the Robertson conditions, J. Math. Phys. 43 (2002), 5183-5222.

[7] Benenti S., Chanu C., Rastelli G., Variable separation theory for the null Hamilton-Jacobi equation, J. Math. Phys. 46 (2005), 042901, 29 pages. 
[8] Błaszak M., Separable bi-Hamiltonian systems with quadratic in momenta first integrals, J. Phys. A: Math. Gen. 38 (2005), 1667-1685. nlin.SI/0312025.

[9] Bolsinov A.V., Matveev V.S., Geometrical interpretation of Benenti systems, J. Geom. Phys. 44 (2003), 489-506.

[10] Chanachowicz M., Chanu C., McLenaghan R.G., Invariant classification of the symmetric $R$-separable webs in $\mathbb{E}_{3}$, in progress.

[11] Chanu C., Rastelli G., Eigenvalues of Killing tensors and orthogonal separable webs, in Proceedings of the International Conference STP2002 "Symmetry and Perturbation Theory" (May 19-24, 2002, Cala Gonone), Editors S. Abenda, G. Gaeta and S. Walcher, World Scietific Publishing, Singapore, 2002, 18-25.

[12] Chanu C., Rastelli G., Fixed energy R-separation for Schrödinger equation, Int. J. Geom. Methods Mod. Phys. 3 (2006), 489-508, nlin.SI/0512033.

[13] Degiovanni L., Rastelli G., Complex variables for separation of Hamilton-Jacobi equation on real pseudoRiemannian manifolds, nlin.SI/0610012.

[14] Degiovanni L., Rastelli G., Complex variables for separation of Hamilton-Jacobi equation on threedimensional Minkowski space, Int. J. Geom. Methods Mod. Phys., to appear, nlin.SI/0612051.

[15] Grigoryev Yu.A., Tsiganov A.V., Symbolic software for separation of variables in the Hamilton-Jacobi equation for the L-systems, Regul. Chaotic Dyn. 10 (2005), 413-422, nlin.SI/0505047.

[16] Kalnins E.G., Separation of variables for Riemannian spaces of constant curvature, Pitman Monographs and Surveys in P. A. Math., Vol. 28, Longman Scientific and Technical, New York, 1986.

[17] Kalnins E.G., Miller W.Jr., Killing tensors and variable separation for the Hamilton-Jacobi and Helmholtz equations, SIAM J. Math. Anal. 11 (1980), 1011-1026.

[18] Kalnins E.G., Miller W.Jr., Conformal Killing tensors and variable separation for HJEs, SIAM J. Math. Anal. 14 (1983), 126-137.

[19] Kalnins E.G., Miller W.Jr., Intrinsic characterisation of orthogonal $R$-separation for Laplace equation, J. Phys. A: Math. Gen. 15 (1982), 2699-2709.

[20] Moon P., Spencer D.E., Field theory handbook, Springer Verlag, Berlin 1961.

[21] Rauch-Wojciechowski S., Waksjö C., How to find separation coordinates for the Hamilton-Jacobi equation: a criterion of separability for natural Hamiltonian systems, Math. Phys. Anal. Geom. 6 (2003), 301-348. 